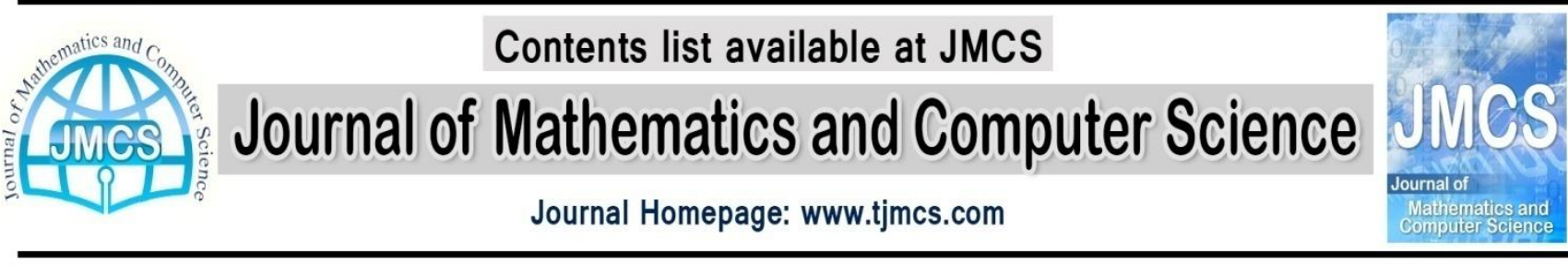

\section{Improvement of the Multiquadric Quasi-Interpolation $L_{W_{2}}$}

\author{
Maryam Sarboland, Azim Aminataei \\ Faculty of Mathematics, Department of Applied Mathematics, K. N. Toosi University of \\ Technology, P.O. Box: 15418-49611, Tehran, Iran \\ sarboland@dena.kntu.ac.ir, ataei@kntu.ac.ir
}

Article history:

Received April 05, 2014

Accepted May 14, 2014

Available online June 2014

\begin{abstract}
In this paper, we improve the multiquadric (MQ) quasi-interpolation operator $L_{W_{2}}$. The operator $L_{W_{2}}$ is based on inverse multiquadric radial basis function (IMQ-RBF) interpolation, and $\mathrm{Wu}$ and Schaback's MQ quasi-interpolation operator $L_{D}$. In definition process of the quasi-interpolation $L_{W_{2}}$, the second derivative of function is used that approximated by center finite difference. In this paper, we use compact finite difference for approximation of the second derivative and increase accuracy of quasi-interpolation $L_{W_{2}}$. Numerical experiments demonstrate that the proposed MQ quasi-interpolation scheme is valid.
\end{abstract}

Keywords: Radial basis function; Multiquadric quasi-interpolation; Inverse multiquadric; Compact finite difference.

\section{Introduction}

Radial basis functions (RBFs) are a tool for interpolating data. Applications of RBFs include bathymetry, topography, hydrology, mapping, geophysics, geology, image warping and medical imaging, and see [1, $4,8,14,15,18,26]$. Experience in a variety of applications has shown RBFs to be particularly well suited to scattered data interpolation problem. RBFs have been widely used as a spatial approximation scheme in various fields such as neural networks and solution of differential equation [3, 5, 9-12, 21-23, 25].

In the RBF interpolation, we have to solve a linear system of equations where the system matrix tend to become very ill-condition as the interpolating data distributed densely. To avoid this problem, the multiquadric (MQ) quasi-interpolation method is suggested. 
MQ quasi-interpolation is constructed directly from linear combination of MQ basis and the approximated function. Hon and $\mathrm{Wu}[17], \mathrm{Wu}[27,28]$ and others have provided some successful examples using MQ quasi-interpolation scheme for solving differential equations. Beatson and Powell [2] and $\mathrm{Wu}$ and Schaback [29] proposed other univariate MQ quasi-interpolations. Recently, Jiang et al. [19] have introduced a new multi-level univariate MQ quasi-interpolation approach with high approximation order compared with initial MQ quasi-interpolation scheme, namely as $L_{W}$ and $L_{W_{2}}$. This approach is based on inverse multiquadric (IMQ) RBF interpolation, and Wu and Schaback's MQ quasi-interpolation operator $L_{D}$ that have the advantages of high approximation order. Up to now, the MQ quasi-interpolation scheme is used for various partial differential equations (PDEs) such as Korteweg-de Vries (KdV), SineGordon and Burgers' equations, see [6, 7, 13, 16, 20,30].

In definition process of the quasi-interpolation $L_{W_{2}}$, the second derivative of function is used that approximated by center finite difference. In this paper, we use compact finite difference for approximation of the second derivative and increase accuracy of quasi-interpolation $L_{W_{2}}$.

The rest of present paper is arranged as follows. Brief information of the MQ quasi-interpolation operators $L_{W}$ and $L_{W_{2}}$ and improvement from $L_{W_{2}}$ are given in Section 2. Several numerical experiments are presented in Section 3, followed by a conclusion summary in Section 4.

\section{The MQ quasi-interpolation scheme}

For a given interval $\Omega=[a, b]$ and a finite set of distinct point

$a=x_{0}<x_{1}<\cdots<x_{N}=b, h=\max _{1 \leq i \leq N}\left(x_{i}-x_{i-1}\right)$,

quasi-interpolation of a univariate function $f:[a, b] \rightarrow \mathbb{R}$ is given by

$L(f)=\sum_{i=0}^{N} f\left(x_{i}\right) \Psi_{i}(x)$

where function $\Psi_{i}(x)$ is a linear combination of the MQs

$\Psi_{i}(x)=\sqrt{c^{2}+\left(x-x_{i}\right)^{2}}$

and $c \in \mathbb{R}^{+}$is a shape parameter. In [29], Wu and Scheback presented the univariate MQ quasi-

interpolation operator $L_{D}$ that is defined as

$L_{D} f(x)=\sum_{i=0}^{N} f\left(x_{i}\right) \widetilde{\Psi}_{i}(x)$

where

$\widetilde{\Psi}_{0}(x)=\frac{1}{2}+\frac{\Psi_{1}(x)-\left(x-x_{0}\right)}{2\left(x_{1}-x_{0}\right)}$,

$\widetilde{\Psi}_{1}(x)=\frac{\Psi_{2}(x)-\Psi_{1}(x)}{2\left(x_{2}-x_{1}\right)}+\frac{\Psi_{1}(x)-\left(x-x_{0}\right)}{2\left(x_{1}-x_{0}\right)}$, 
$\widetilde{\Psi}_{i}(x)=\frac{\Psi_{i+1}(x)-\Psi_{i}(x)}{2\left(x_{i+1}-x_{i}\right)}+\frac{\Psi_{i}(x)-\Psi_{i-1}(x)}{2\left(x_{i}-x_{i-1}\right)}, \quad 2 \leq i \leq N-2$

$\widetilde{\Psi}_{N-1}(x)=\frac{\left(x_{N}-x\right)-\Psi_{N-1}(x)}{2\left(x_{N}-x_{N-1}\right)}+\frac{\Psi_{N-1}(x)-\Psi_{N-2}(x)}{2\left(x_{N-1}-x_{N-2}\right)}$,

and

$\widetilde{\Psi}_{N}(x)=\frac{1}{2}+\frac{\Psi_{N-1}(x)-\left(x_{N}-x\right)}{2\left(x_{N}-x_{N-1}\right)}$.

In RBFs interpolation, high approximation order can be gotten by increasing the number of interpolation centers but we have to solve unstable linear system of equations. By using MQ quasiinterpolation scheme, we can avoid this problem, whereas the approximation order is not good. Therefore, Jiang et al. [19] defined two MQ quasi-interpolation operators denoted as $L_{W}$ and $L_{W_{2}}$ which pose the advantages of RBFs interpolation and MQ quasi-interpolation scheme. The process of MQ quasiinterpolation of $L_{W}$ and $L_{W_{2}}$ are as follows that is described in [19].

Suppose that $\left\{x_{k_{i}}\right\}_{i=1}^{\bar{N}}$ is a smaller set from the given points $\left\{x_{i}\right\}_{i=0}^{N}$ where $\bar{N}$ is a positive integer satisfying $\bar{N}<N$ and $0=k_{0}<k_{1}<\cdots<k_{\bar{N}+1}=N$. Using the IMQ-RBF, the second derivative of $f(x)$ can be approximated by RBF interpolant $S_{f}$ as

$S_{f^{\prime \prime}}(x)=\sum_{j=1}^{\bar{N}} \alpha_{j} \bar{\varphi}_{j}(x)$

where

$\bar{\varphi}_{j}(x)=\frac{s^{2}}{\left(s^{2}+\left(x-x_{k_{i}}\right)^{2}\right)^{3 / 2}}$

and $s \in \mathbb{R}^{+}$is a shape parameter. The coefficients $\left\{\alpha_{j}\right\}_{j=1}^{\bar{N}}$ are uniquely determined by the interpolation condition

$S_{f^{\prime \prime}}\left(x_{k_{i}}\right)=\sum_{j=1}^{\bar{N}} \alpha_{j} \bar{\varphi}_{j}\left(x_{k_{i}}\right)=f^{\prime \prime}\left(x_{k_{i}}\right), \quad 1 \leq i \leq \bar{N}$.

Since, the Eq. (4) is solvable [24], so

$\alpha=A_{X}^{-1} \cdot f_{X}^{\prime \prime}$

where

$X=\left\{x_{k_{1}}, \ldots, x_{k_{\bar{N}}}\right\}, \quad \alpha=\left[\alpha_{1}, \ldots, \alpha_{\bar{N}}\right]^{T}, \quad A_{X}=\left[\bar{\varphi}_{j}\left(x_{k_{i}}\right)\right], \quad f_{X}^{\prime \prime}=\left[f^{\prime \prime}\left(x_{k_{1}}\right), \ldots, f^{\prime \prime}\left(x_{k_{\bar{N}}}\right)\right]^{T}$.

By using $f$ and the coefficient $\alpha$ defined in Eq. (12), a function $e(x)$ is constructed in the form

$e(x)=f(x)-\sum_{i=1}^{\bar{N}} \alpha_{i} \sqrt{s^{2}+\left(x-x_{k_{i}}\right)^{2}}$.

Then the MQ quasi-interpolation operator $L_{W}$ by using $L_{D}$ defined by Eqs. (1) and (2) on the data 
$\left(x_{i}, e\left(x_{i}\right)\right)_{0 \leq i \leq N}$ with the shape parameter $c$ is defined as follows:

$L_{W} f(x)=\sum_{i=1}^{\bar{N}} \alpha_{i} \sqrt{s^{2}+\left(x-x_{k_{i}}\right)^{2}}-L_{D} e(x)$.

The shape parameters $c$ and $s$ should not be the same constant in Eq. (7).

In Eq. (4), $f_{x_{k_{i}}}^{\prime \prime}$ can be replaced by

$f_{x_{k_{i}}}^{\prime \prime}=\frac{f\left(x_{k_{i+1}}\right)-2 f\left(x_{k_{i}}\right)+f\left(x_{k_{i-1}}\right)}{h_{2}^{2}}$, with $h_{2}=\frac{b-a}{\bar{N}}$

when the data $\left(x_{k_{i}}, f\left(x_{k_{i}}\right)\right)_{0 \leq i \leq \bar{N}}$ are given, and $\left(x_{i}\right)_{0 \leq i \leq N}$ are equally spaced points. So, if $f_{X}^{\prime \prime}$ in Eq. (12) replace by

$F_{X}^{\prime \prime}=\left(f_{x_{k_{1}}}^{\prime \prime}, \ldots, f_{x_{k_{\bar{N}}}}^{\prime \prime}\right)^{T}$

the quasi-interpolation operator defined by Eqs. (6) and (7) is denoted by $L_{W_{2}}$. For more details about the properties and accuracy of $L_{W}$ and $L_{W_{2}}$, one can see [19].

If $f^{\prime \prime}\left(x_{k_{i}}\right)$ in Eq. (3) replace by

$f^{\prime \prime}\left(x_{k_{i}}\right)=\frac{\delta_{x}^{2}}{h_{2}^{2}\left(1+12 \delta_{x}^{2}\right)} f\left(x_{k_{i}}\right)$,

where $\delta_{x}^{2} f\left(x_{k_{i}}\right)=f\left(x_{k_{i+1}}\right)-2 f\left(x_{k_{i}}\right)+f\left(x_{k_{i-1}}\right)$ yields

$\sum_{j=1}^{\bar{N}} \alpha_{j} \bar{\varphi}_{j}\left(x_{k_{i}}\right)=\frac{\delta_{x}^{2}}{h_{2}^{2}\left(1+12 \delta_{x}^{2}\right)} f\left(x_{k_{i}}\right), \quad 1 \leq i \leq \bar{N}$.

At the result, the coefficients $\left\{\alpha_{i}\right\}_{i=1}^{\bar{N}}$ are uniquely determined by the linear system

$\alpha=A_{X}^{*}{ }^{-1} \cdot f_{X}^{\prime \prime}$

where $A_{X}^{*}=\left[\left(1+12 \delta_{x}^{2}\right) \bar{\varphi}_{j}\left(x_{k_{i}}\right)\right]$. In this case, the quasi-interpolation operator defined in Eq. (7) is denoted by $L_{W_{2}}$.

\section{The numerical experiments}

Three experiments are studied to investigate the robustness and the accuracy of the proposed method. The numerical results of the quasi-interpolation $L_{W_{2}}$ is compared with these associated with $L_{W}$ and $L_{W_{2}}$. The $L_{\infty}$ norm of $2^{12}$ equally spaced points on $[0,1]$ which is defined by

$L_{\infty}=\|L f-f\|_{\infty}=\max _{0 \leq j \leq 2^{12}}\left|L f\left(\xi_{j}\right)-f\left(\xi_{j}\right)\right|$, 
is used to measure the accuracy of the schemes. In all experiments, the shape parameters $s$ and $c$ are considered as $10 h_{2}$ and $h$, respectively, and $h_{2}=4 h$.

The computations associated with our experiments are performed in Maple 14 on a PC with a CPU of 2.4 GHZ.

\section{Experiment 1}

In this experiment, we consider the function

$f_{1}(x)=\sin (4.5 x)$

The $L_{\infty}$ error of the approximated results by using $L_{\bar{W}_{2}}$ is listed in Table 1 and compared with the quasiinterpolation operators $L_{W}$ and $L_{W_{2}}$. The graphs of $f_{1}(x)$ and $L_{\infty}$ error approximation of $f_{1}(x)$ by using $L_{\bar{W}_{2}}, L_{W}$ and $L_{W_{2}}$ are given in Fig. 1.

Table 1 shows that the scheme $L_{\bar{W}_{2}}$ is more accurate than $L_{W}$ and $L_{W_{2}}$ schemes.
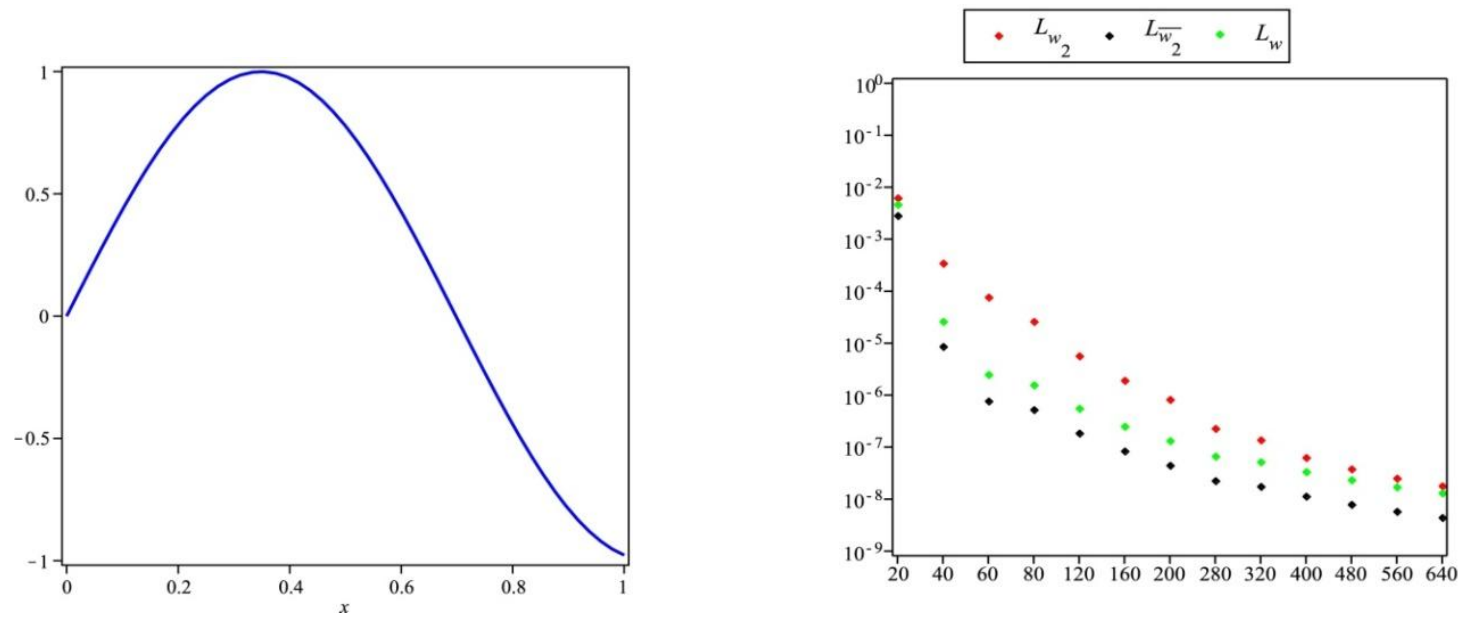

Figure 1: The graphs of $f_{1}(x)$ (left) and error approximation of $f_{1}(x)$ (right) by using $L_{W_{2}}, L_{W}$ and $L_{W_{2}}$ of experiment 1 .

Table 1: The $L_{\infty}$ of the MQ quasi-interptolation $L_{\bar{W}_{2}}, L_{W}$ and $L_{W_{2}}$ with different number of data points of experiment 1 .

\begin{tabular}{cccc}
\hline$N$ & $\boldsymbol{L}_{\bar{W}_{2}}$ & $\boldsymbol{L}_{W}$ & $\boldsymbol{L}_{W_{2}}$ \\
\hline 40 & $8.73351 \times 10^{-6}$ & $2.64131 \times 10^{-5}$ & $3.49141 \times 10^{-4}$ \\
80 & $5.31134 \times 10^{-7}$ & $1.59019 \times 10^{-6}$ & $2.64502 \times 10^{-5}$ \\
160 & $8.51048 \times 10^{-8}$ & $2.54117 \times 10^{-7}$ & $1.94118 \times 10^{-6}$ \\
320 & $1.77623 \times 10^{-8}$ & $5.25990 \times 10^{-8}$ & $1.39288 \times 10^{-7}$ \\
640 & $4.46403 \times 10^{-9}$ & $1.32682 \times 10^{-8}$ & $1.81210 \times 10^{-8}$ \\
\hline
\end{tabular}




\section{Experiment 2}

In this experiment, the following function

$f_{2}(x)=x^{9}$

is considered. The $L_{\infty}$ error of the approximated results by using $L_{W_{2}}$ is listed in Table 2 and compared with the quasi-interpolation operators $L_{W}$ and $L_{W_{2}}$. The profile of the function and errors of the approximating results by using $L_{\bar{W}_{2}}, L_{W}$ and $L_{W_{2}}$ are shown in Fig. 2.

\section{Experiment 3}

In this experiment, we consider the function

$f_{3}(x)=\sin (x)+0.1 \sin (32 x)$.

The graphs of $f_{3}(x)$ and $L_{\infty}$ error of numerical results by using all of the MQ quasi-interpolations mentioned in this paper for this function are shown in Fig. 3.
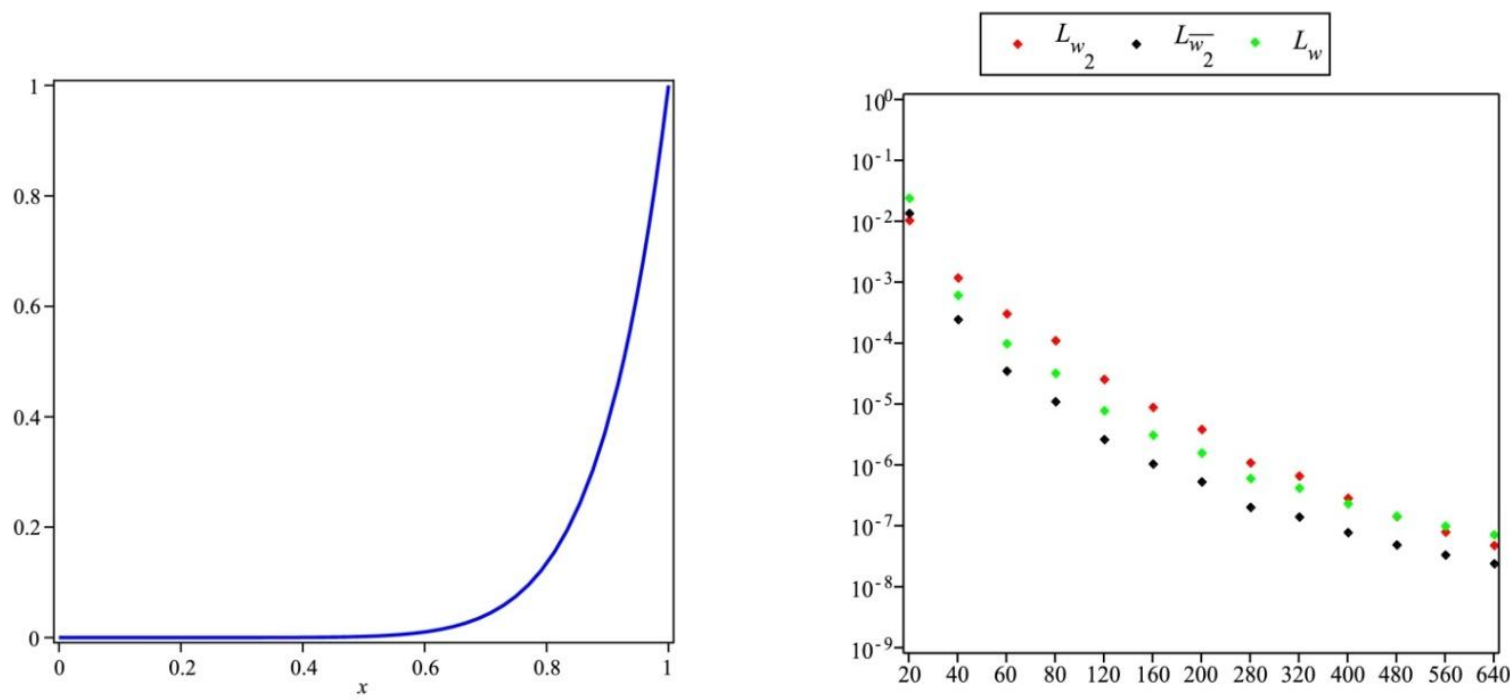

Figure 2: The graphs of $f_{2}(x)$ (left) and error approximation of $f_{2}(x)$ (right) by using $L_{\bar{W}_{2}}, L_{W}$ and $L_{W_{2}}$ of experiment 2.

Table 2: The $L_{\infty}$ of the MQ quasi-interpolation $L_{\bar{W}_{2}}, L_{W}$ and $L_{W_{2}}$ with different number of data points of experiment 2.

\begin{tabular}{cccc}
\hline$N$ & $\boldsymbol{L}_{\bar{W}_{2}}$ & $\boldsymbol{L}_{W}$ & $\boldsymbol{L}_{W_{2}}$ \\
\hline 40 & $2.50206 \times 10^{-4}$ & $6.24470 \times 10^{-4}$ & $1.20701 \times 10^{-3}$ \\
80 & $1.12242 \times 10^{-5}$ & $3.27713 \times 10^{-5}$ & $1.12681 \times 10^{-4}$ \\
160 & $1.06403 \times 10^{-6}$ & $3.16585 \times 10^{-6}$ & $9.03069 \times 10^{-6}$ \\
320 & $1.43486 \times 10^{-7}$ & $4.26968 \times 10^{-7}$ & $6.74010 \times 10^{-7}$ \\
640 & $2.45344 \times 10^{-8}$ & $7.30686 \times 10^{-8}$ & $4.88460 \times 10^{-8}$ \\
\hline
\end{tabular}



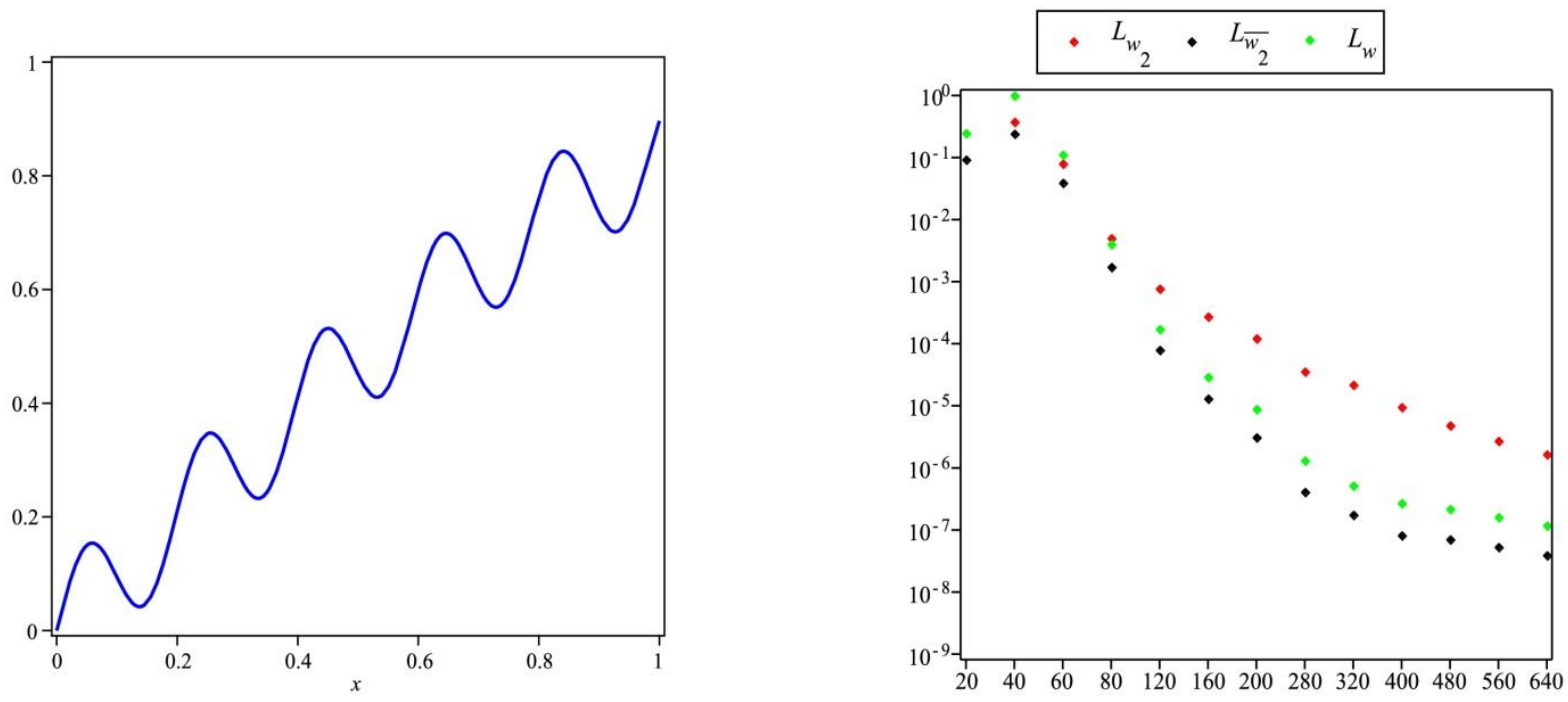

Figure 3: The graphs of $f_{3}(x)$ (left) and error approximation of $f_{3}(x)$ (right) by using $L_{\bar{W}_{2}}, L_{W}$ and $L_{W_{2}}$ of experiment 3.

Table 3: The $L_{\infty}$ of the MQ quasi-interpolation $L_{\bar{W}_{2}}, L_{W}$ and $L_{W_{2}}$ with different number of data points of experiment 3.

\begin{tabular}{cccc}
\hline$N$ & $\boldsymbol{L}_{\bar{W}_{\mathbf{2}}}$ & $\boldsymbol{L}_{\boldsymbol{W}}$ & $\boldsymbol{L}_{\boldsymbol{W}_{2}}$ \\
\hline 40 & $2.43413 \times 10^{-1}$ & $1.00499 \times 10^{-0}$ & $3.79953 \times 10^{-1}$ \\
80 & $1.73800 \times 10^{-3}$ & $4.05360 \times 10^{-3}$ & $5.06065 \times 10^{-3}$ \\
160 & $1.31477 \times 10^{-5}$ & $2.92361 \times 10^{-5}$ & $2.76056 \times 10^{-4}$ \\
320 & $1.76708 \times 10^{-7}$ & $2.22275 \times 10^{-7}$ & $2.19455 \times 10^{-5}$ \\
640 & $3.93651 \times 10^{-8}$ & $1.18591 \times 10^{-7}$ & $1.66290 \times 10^{-6}$ \\
\hline
\end{tabular}

Also, the approximated results by using $L_{W_{2}}$ is listed in Table 3 and compared with the quasi-interpolation operators $L_{W}$ and $L_{W_{2}}$. Table 3 shows that the scheme $L_{\bar{W}_{2}}$ is more accurate than $L_{W}$ and $L_{W_{2}}$ schemes.

\section{Conclusion}

In this paper, a MQ quasi-interpolation $L_{\bar{W}_{2}}$ based on Jiang et al. [16] MQ quasi-interpolation scheme and compact finite difference scheme is presented. The numerical results which are given in the previous section demonstrate the good accuracy of the present scheme. Also, the Tables show that this scheme performs better than $L_{W}$ and $L_{W_{2}}$ methods.

In the present method, we have to use equidistant data that it is a weakness of the method whereas $L_{W}$ scheme can be used for non-equidistant data. Moreover, by using the following value 
$f_{x_{k_{i}}}^{\prime \prime}=\frac{2\left[\left(x_{k_{i}}-x_{k_{i-1}}\right) f\left(x_{k_{i+1}}\right)-\left(x_{k_{i+1}}-x_{k_{i-1}}\right) f\left(x_{k_{i}}\right)+\left(x_{k_{i+1}}-x_{k_{i}}\right) f\left(x_{k_{i-1}}\right)\right]}{\left(x_{k_{i}}-x_{k_{i-1}}\right)\left(x_{k_{i+1}}-x_{k_{i}}\right)\left(x_{k_{i+1}}-x_{k_{i-1}}\right)}$

instead of Eq. (8), $L_{W_{2}}$ can also be applied for scattered data.

\section{References}

[1] I. Barrodale, D. Skea, M. Berkley, R. Kuwahara, P. Poeckert, Warping digital images using thin-plate splines, Pattern Recognition 26 (1993) 375-376.

[2] R.K. Beatson, M.J.D. Powell, Univariate multiquadric approximation: quasi-interpolation to scattered data, Constr. Approx. 8 (1992) 275-288.

[3] J. Biazar, M. Hosami, Two efficient approaches based on radial basis functions to nonlinear time-dependent partial differential equations, J. Math. Computer Sci. 13 (2014) 1-11.

[4] J.C. Carr, W.R. Fright, R.K. Beatson, Surface interpolation with radial basis functions for medical imaging, IEEE Trans. Medical Imaging 16 (1997) 96-107.

[5] Y.L. Chan, L.H. Shen, C.T. Wu, D.L. Young, A novel upwind-based local radial basis function differential quadrature method for convection-dominated flows, Computer \& Fluids 89 (2014) 157-166.

[6] R.H. Chen, Z.M. Wu, Solving partial differential equation by using multiquadric quasi-interpolation, Appl. Math. Comput. 186 (2007) 1502-1510.

[7] R.H. Chen, Z.M. Wu, Solving hyperbolic conservation laws using multiquadric quasi-interpolation, Numer. Methods Partial Differential Equations 22 (2006) 776-796.

[8] B.H. Chovitz, Geodetic theory, Rev. geophys. Space Phys. 13 (1975) 243-245.

[9] M. Dehghan, A. Shokri, A numerical method for solution of the two-dimensional Sine-Gordon equation using the radial basis functions, Mathematics and Computers Simulation 79 (2008) 700-715.

[10] M. Dehghan, A. Nikpour, Numerical solution of the system of second-order boundary value problems using the local radial basis function based differential quadrature collocation method, Appl. Math. Modelling, 37 (2013) 8578-8599.

[11] M. Dehghan, A. Shokri, Numerical solution of the nonlinear Klein-Gordon equation using radial basis functions, J. Comput. Appl. Math. 230 (2009) 400-410.

[12] M. Dehghan, A. Shokri, A numerical method for KdV equation using collocation and radial basis functions, Nonlinear Dyn. 50 (2007) 111-120.

[13] F. Gao, Ch. Chi, Numerical solution of nonlinear Burgers' equation using high accuracy multiquadric quasi-interpolation, Appl. Math. Comput. 229 (2014) 414-421.

[14] R.L. Hardy, S.A. Nelson, A multiquadric biharmonic representation and approximation of disturbing potential, Geophys. Res. Lett. 13 (1986) 18-21.

[15] R.L. Hardy, Theory and applications of the multiquadric biharmonic method, Comput. Math. Appl. 19 (1990) $163-208$.

[16] Y.C. Hon, X.Z. Mao, An efficient numerical scheme for Burgers' equation, Appl. Math. Comput. 95 (1998) 37-50.

[17] Y.C. Hon, Z.M. Wu, A quasi-interpolation method for solving stiff ordinary differential equations, Internat. J. Numer. Methods Eng. 48 (2000) 1187-1197. 
[18] J.R. Jancaitis, J. L. Junkins, Modeling irregular surfaces, Photogramm. Engng. 39 (1973) 413-420.

[19] Z.W. Jiang, R.H. Wang, C.G. Zhu, M. Xu, High accuracy multiquadric quasi-interpolation, Appl. Math. Modelling 35 (2011) 2185-2195.

[20] Z.W. Jiang, R.H. Wang, Numerical solution of one-dimensional Sine-Gordon equation using high accuracy multiquadric quasi-interpolation, Appl. Math. comput. 218 (2012) 7711-7716.

[21] E.J. Kansa, Multiquadric-a scattered data approximation scheme with applications to computational fluid dynamics I, Comput. Math. Appl. 19 (1990) 127-145.

[22] E.J. Kansa, Multiquadric-a scattered data approximation scheme with applications to computational fluid dynamics II, Comput. Math. Appl. 19 (1990) 147-161.

[23] M. Kumar, N. Yadav, Multilayer perceptrons and radial basis function neural network methods for the solution of differential equations: A survey, Comput. Math. With Applications 62 (2011) 3796-3811.

[24] W.R. Madych and S.A. Nelson, Multivariate interpolation and conditionally positive defnite functions, Math. Comp. 54 (1990) 211-230.

[25] K. Parand, S. Abbasbandy, S. Kazem, J.A. Rad, A novel application of radial basis functions for solving a model of firstorder integro-ordinary differential equation, Communications in Nonlinear Science and Numerical Simulation 16 (2011) 4250-4258.

[26] D.T. Sandwell, Biharmonic spline interpolation of GEOS-3 and SEASAT altimeter data, Geophys. Res. Lett. 14 (1987) 139-142.

[27] Z.M. Wu, Dynamically knots setting in meshless method for solving time dependent propagations equation, Comput.Methods Appl. Mech. Eng. 193 (2004) 1221-1229.

[28] Z.M. Wu, Dynamically knot and shape parameter setting for simulating shock wave by using multiquadric quasiinterpolation, Engineering Analysis with Boundary Elements 29 (2005) 354-358.

[29] Z.M. Wu, R. Schaback, Shape preserving properties and convergence of univariate multiquadric quasi- interpolation, Acta. Math. Appl. Sin. Engl. Ser. 10 (1994) 441-446.

[30] M.L. Xiao, R.H. Wang, C.H. Zhu, Applying multiquadric quasi-interpolation to solve KdV equation, Mathematical Research Exposition 31 (2011) 191-201. 